\title{
APPLICABILITY OF ELISA METHODS FOR HIGH GLUTEN-CONTAINING SAMPLES
}

\author{
E. Schall, Zs. Bugyi, L. Hajas, K. TöRÖK and S. TömÖSKÖZi* \\ Department of Applied Biotechnology and Food Science, Faculty of Chemical Technology and Biotechnology, \\ Budapest University of Technology and Economics, H-1111 Budapest, Szent Gellért tér 4. Hungary
}

(Received: 19 December 2018; accepted: 1 February 2019)

Quantitation of gluten in gluten-free products is a great challenge as it is hindered by several factors including the lack of certified reference materials. To resolve this problem, our research group, in cooperation with other international experts, started a series of experiments with the goal of the production of a suitable gluten reference material. As a part of this research, several different wheat cultivars and their isolated gluten proteins were characterized by different methods, including enzyme-linked immunosorbent assay (ELISA). However, we need to know the performance of the ELISA methods used for this special area of research. During the present work we investigated the accuracy and precision of two different ELISA methods for our own laboratory conditions and special sample matrices (wheat flours and gliadin isolate). We have found that the tested performance characteristics of the methods seem to be appropriate on a case-by-case basis, but the long-term measurement uncertainty is higher, which makes it difficult to evaluate the results obtained with the ELISA method for these types of samples.

Keywords: celiac disease, gluten, ELISA, method performance, reference material

Beside their key role in the formation of unique technological behaviour of wheat-based flours and bakery products, gluten proteins of wheat (and also rye and barley) can trigger celiac disease in about $1 \%$ of the population. The only treatment is a lifelong gluten-free diet (Ludvigsson et al., 2013). According to Codex Alimentarius, gluten free products cannot contain more than $20 \mathrm{mg} \mathrm{kg}^{-1}$ gluten (CODEX STANDARD 118-1979, 2015). Various analytical methods are available for determination or checking the gluten content, such as enzymelinked immunosorbent assay (ELISA), liquid chromatography with mass spectrometry detection, or molecular biological techniques (e.g. polymerase chain reaction). However, the most commonly used method in routine analysis is ELISA. These methods are based on immunochemical reactions, in which antibodies recognize one or more short peptide sequences (epitopes) of gluten proteins. This reaction results in a very specific and sensitive determination (SCHERF \& POMS, 2016). Commercially available ELISA tests provide partially different strategies for determining gluten concentration as they apply different antibodies, extraction procedures, and calibrating materials (DiAz-Amigo \& Popping, 2013). Consequently, ELISA tests can give different results for the same sample (BugYi et al., 2013; SCHERF, 2017). The determination of gluten is further complicated, because the protein content and composition of cereals vary depending on genetic (species, varieties) and environmental factors (harvest year and agricultural practices). As a result, these factors also affect the relative amount of epitopes that are recognized by antibodies commonly used in gluten ELISA test kits (HAJAS et al., 2018; SCHOPF \& SCHERF, 2018). Complexity of the food matrices

\footnotetext{
* To whom correspondence should be addressed.

Phone: +36 1463 1419; fax: +36 1463 3855; e-mail: tomoskozi@mail.bme.hu
}

0139-3006 (C) 2019 The Author(s) 
and the effects of food processing on the target proteins are also restraining factors of gluten analysis (BESLER et al., 2001; TÖRÖK et al., 2015).

Another major difficulty of this field is the lack of certified reference materials (DiAzAmigo \& Popping, 2013; HajAs et al., 2018). Today, the widely used standard-like material for gluten analysis is the PWG-gliadin. It is an isolated protein fraction from 28 European wheat cultivars with good solubility, homogeneity, and stability properties (VAN ECKERT et al., 2006). It was proposed for approval as a certified reference material (RM), but it does not meet some of the RM requirements, such as reproducibility of production (Diaz-Amigo \& Popping, 2013).

In an international framework, our group has put a lot of effort into gluten RM development (HAJAS et al., 2016, 2018). The speciality of our RM development work is that we had to investigate different matrices with high gluten protein contents, like wheat flours, gluten or gliadin isolates. As these types of samples are not included in the intended use of the ELISA methods, it is very important to understand their performance characteristics for this application. The presented work can be considered as an in-house validation of the methods for the examined sample matrices. By establishing the performance, it is possible to include ELISA methods for gluten RM development purposes.

\section{Materials and methods}

\subsection{Experimental design}

Several factors were studied to assess the performance of ELISA methods. The effect of the applied methods (including different sample preparations, epitopes, and reagents) was examined by using two different ELISA methods. The matrix effect was investigated using pure protein isolate, which contains just the target protein fractions (gliadins) including proteins with target epitopes, and two wheat flours containing all protein fractions (gluten proteins, albumins, and globulins), other macromolecules (starch, lipids, and fibres), and minor ingredients. The effect of kit stability was studied by the utilisation of kits with different LOT numbers. The effect of plate homogeneity was investigated by placing the samples on the same strips and randomly on the ELISA plates. The in-house repeatability was investigated by the evaluation of the results of two operators (marked with " $\mathrm{X}$ " and "Y"). For the determination of accuracy, gliadin recovery values were calculated with the evaluation of results obtained by reversed-phase high-performance liquid chromatography (RP-HPLC) method. The stability of samples was studied by using different results, which were performed on a total of 20 measurement days for a period of approximately 2.5 years.

\subsection{Samples}

Two wheat (Triticum aestivum L.) cultivars (Capo from Austria and Glenlea from Canada) were used for producing laboratory flour samples from the harvest year of 2014. PWG gliadin was used as a pure isolate, and it was also applied in the calibration of the RP-HPLC method (VAN ECKERT et al., 2006). 


\subsection{Production of wheat flours}

Seed moisture content was determined by InfratecTM 1241 Grain Analyser (Foss Tecator AB, Höganäs, Sweden). Wheat samples were conditioned prior to milling according to the Hungarian Standard MSZ 6367-9:1989. Then kernels were milled on a micromill (FQC 109, Metefém, Budapest, Hungary), and the whole meal was sieved $(250 \mu \mathrm{m})$ for $20 \mathrm{~min}$ (AS 200 basic, Retsch GmbH, Haan, Germany).

\subsection{Determination of gliadin content by RP-HPLC}

Wheat flours $(100 \mathrm{mg})$ were extracted sequentially according to the modified Osborne procedure (WIESER et al., 1998) by magnetic stirring with salt solution (extraction of albumins/ globulins), followed by $60 \%$ (v/v) ethanol solution (extraction of gliadins). Suspensions were centrifuged $(3550 \times \mathrm{g}, 25 \mathrm{~min})$, then filtrated. The conditions for the RP-HPLC analyses were the following: instrument: Jasco XLC with Jasco Chrompass Chromatography Data System (Jasco, Pfungstadt, Germany); column: AcclaimTM $300 \mathrm{C} 18$ (particle size $3 \mathrm{~mm}$, pore size $30 \mathrm{~nm}, 2.1 \times 150 \mathrm{~mm}$, Thermo Fisher Scientific, Braunschweig, Germany); detection: UV absorbance at $210 \mathrm{~nm}$.

\subsection{Gliadin quantification with ELISA methods}

The gliadin quantification was performed with two commercially available ELISA test kits: the AgraQuant Gluten G12 Assay (COKAL0200, Romer Labs, Tulln, Austria) and the RIDASCREEN Gliadin Assay (R7001, R-Biopharm, Darmstadt, Germany). They apply different antibodies (monoclonal G12 and monoclonal R5, respectively) and are calibrated differently. The ELISA test kits used for analysis were randomly coded with capital letters ("A" and "B") in the article. ELISA procedures were carried out according to the kit instructions with a few modifications. Three parallel extractions were performed for each sample. The amount of sample to be weighed was reduced 10 times in case of flours and 100 times in case of PWG gliadin. Further dilution was applied with $60 \%$ (v/v) ethanol solution from the clear supernatants: 1:1000 in two steps in the case of flours and 1:2000 in two steps in the case of PWG gliadin. The samples were prepared and diluted independently with freshly prepared reagents for each measurement day. The absorbances were determined using a microplate reader (iMarkTM Microplate Absorbance Reader, Bio-Rad, Hercules, CA, USA). The gliadin/gluten concentration was calculated from the absorbance values by the Bio-Rad Microplate Manager 6 software (Bio-Rad, Hercules, CA, USA) using the curve fit option recommended by the manufacturers (cubic spline in case of method " $A$ " and point-topoint in case of method "B").

\subsection{Statistical analysis}

The analytical results were statistically evaluated with the investigation of means and relative standard deviations. Statistical analysis was carried out by one-sample $t$-test, paired-sample $t$-test, and analysis of variance (ANOVA) at a confidence level of 0.95 using STATISTICA v13 software (TIBCO Software Inc). Thirty-eight independent measurements were analysed for the statistical analysis, 26 of them were measured by method " $\mathrm{A}$ " and 12 by method " $\mathrm{B}$ ". The distribution of the samples and the operators is shown in Figure 1. 


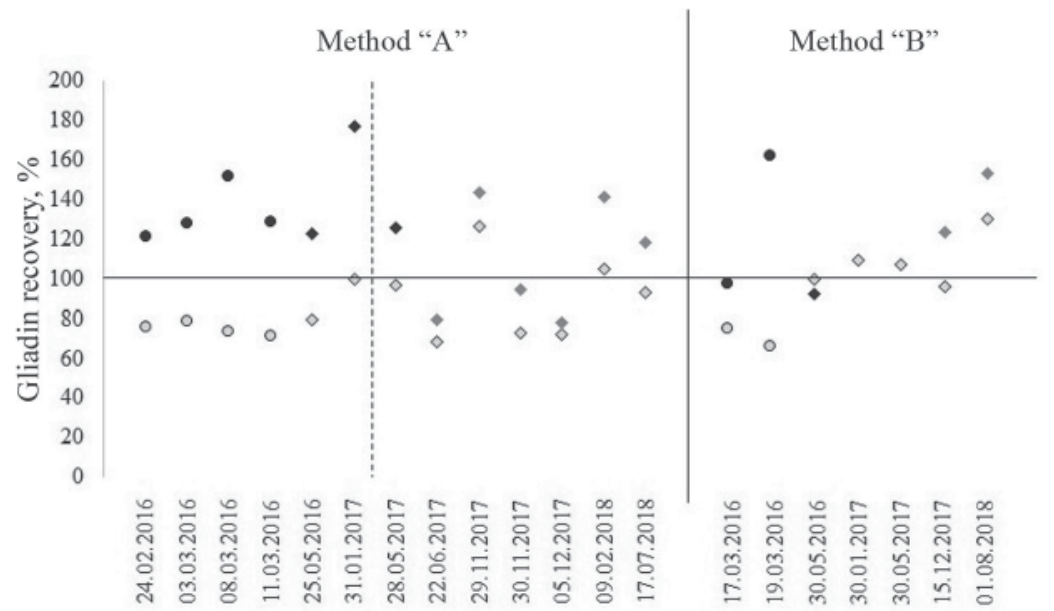

Fig. 1. Gliadin recovery values of the three samples as a function of the measurement day measured by both methods (values before the dotted line were measured by ELISA kits with the same LOT number)

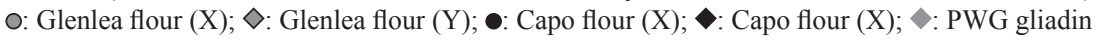

\section{Results and discussion}

\subsection{General assessment of the results}

Based on our gliadin recovery and repeatability values, the accuracy and the precision of both methods are acceptable, because the mean of the gliadin recovery values do not differ significantly from $100 \%$ and the repeatability values are sufficiently low (Table 1/Columns 2 and 4) (Аввотт et al., 2010). Similarly, good gliadin recovery and repeatability values are obtained for the operator-dependent results (Table 1/Columns 6 and 8). However, the average results are paired with great deviation (Table 1/Column 3 ), so the uncertainty of recovery is high. The average recovery values of the different samples are significantly different from $100 \%$ in most cases (Table 1/Column 10) and have a high degree of uncertainty as their values vary from measurement day to day (Fig. 1). We must know if this is the uncertainty of the method, or can we identify factors that affect the performance of ELISA methods? We determined and compared the extents of these effects and tried to identify the possible causes, as well.

\subsection{Examining factors influencing recovery values}

Comparing the average recovery values obtained with the two methods, only $4 \%$ difference is found (Table 1/Column 2). The variation between the operators within method " $\mathrm{A}$ " is only $1 \%$, while in case of method " $\mathrm{B}$ ", it is $14 \%$ (Table $1 /$ Column 6 ). The comparison of the average gliadin recovery values obtained for the different samples are shown in Figure 1 . The Glenlea flour results measured by method " $A$ " are typically less than $100 \%$, and the Capo flour results are higher than $100 \%$. The values of PWG gliadin vary around $100 \%$. In case of method "B", both flours fluctuate around $100 \%$, while PWG gliadin is higher than $100 \%$. The uncertainty of recovery values measured by " $\mathrm{X}$ " is typically lower than measured by "Y" operator (Table 1/Column 11). However, the wide range of deviation for "Y" could be explained by the fact that measurements were made within a much wider time interval than in the case of "X" operator. On the other hand, operator " $X$ " used ELISA kits with the same LOT number in case of method " $A$ ", so the difference in LOT numbers could also affect the results. 


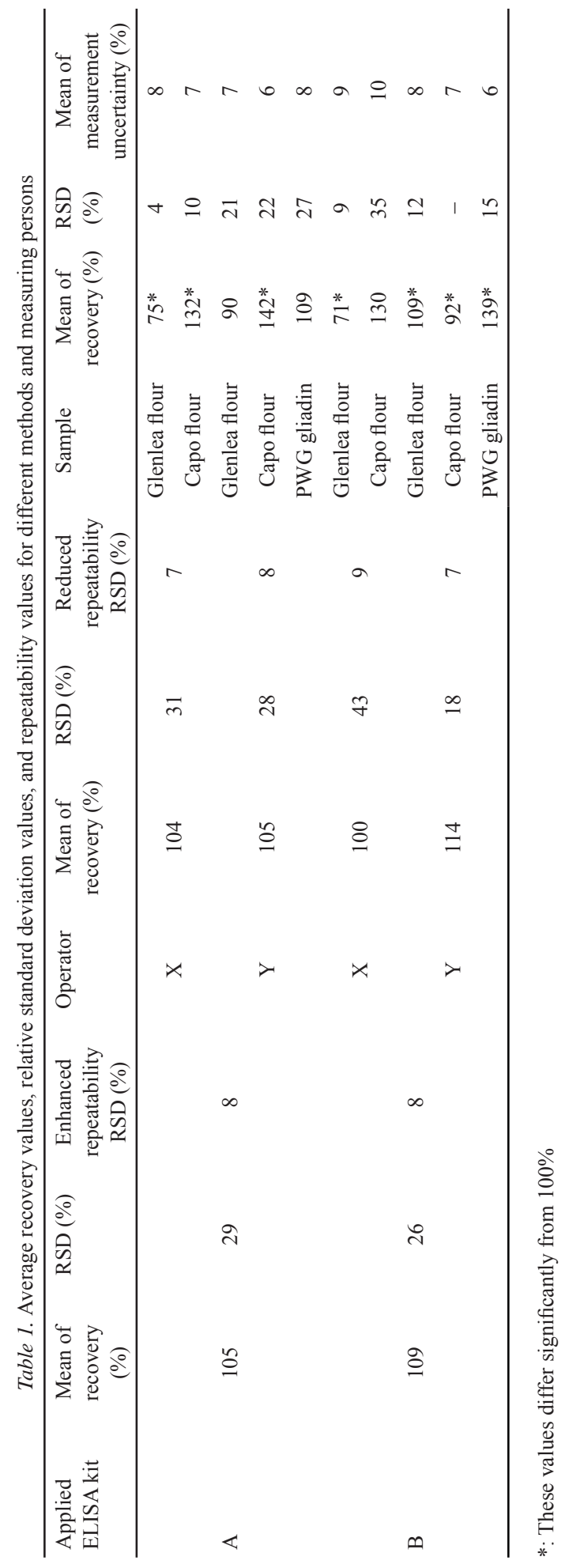

Acta Alimentaria 48, 2019 
Theoretically, the relatively high fluctuation of the recovery values may also be due to the dependence of the results on sample position. The reasons can be a small inhomogeneity of antibody covered wells on ELISA plates or the time necessary for reading the absorbance (in case of higher sample number). The results of randomly and not randomly placed samples are shown in Figure 2. Only small and not significant differences are observed between the two placement modes, so the placement has no effect on the recovery values.

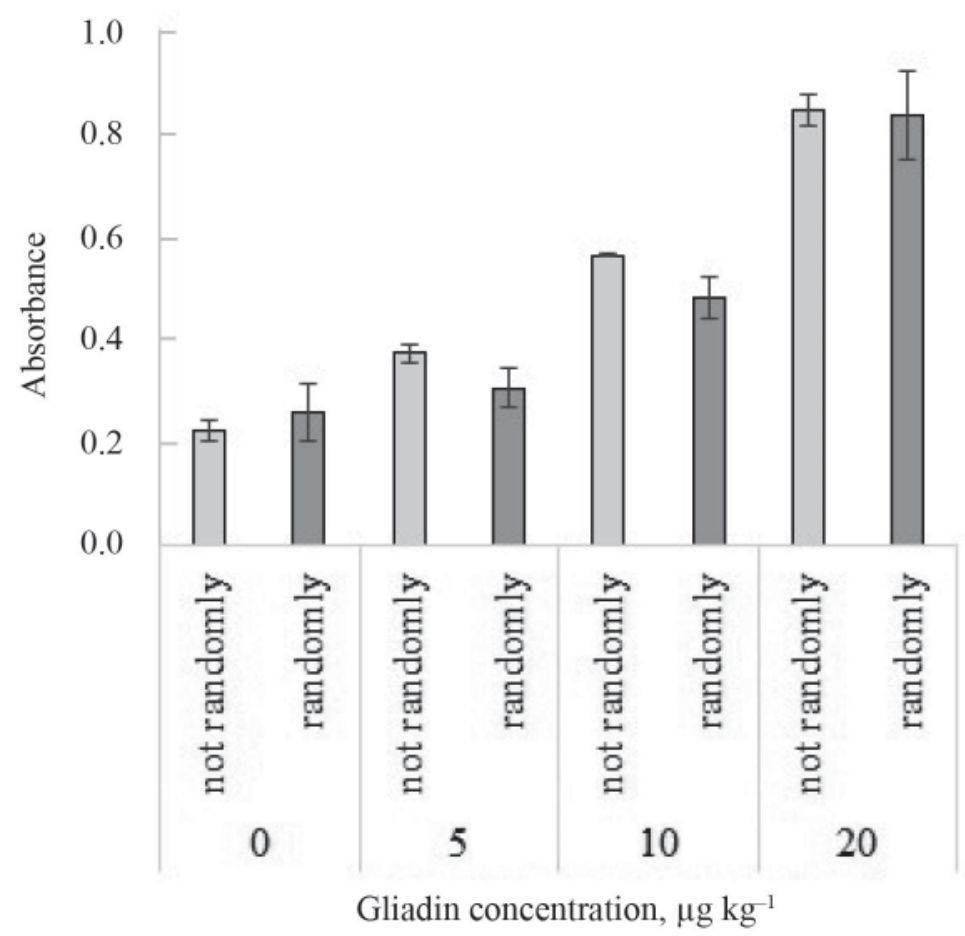

Fig. 2. Absorbance values of standard solutions with $0,5,10$, and $20 \mu \mathrm{g} \mathrm{kg}^{-1}$ gliadin concentrations placed not randomly and randomly on ELISA plate

Comparing the contribution of all factors to the uncertainty of gliadin recovery with analysis of variance, we have found that only the different samples and measuring on different days have a significant effect on the results, which also interact with each other (Fig. 3). It is important to note that the effects of the other factors might not be detected, because these two effects cause very high measurement uncertainty. The different results of the samples can be explained by the presence of the amount of epitopes in them, but there is still no explanation for the differences between the measurement days. It might be caused by change in the stability of the samples, as their properties may change over time. But any positive or negative tendency in the recovery values of all samples cannot be identified through the two and a half year research period (Fig. 1), so it is not likely that sample instability stands behind these observations.

Continuing the investigation of the effects of days, a critical point of the quantitative analysis can be the correct calibration procedures. Figures 4 and 5 show the calibration curves of the ELISA kit " $\mathrm{A}$ " and " $\mathrm{B}$ " on each measurement day. It is clear that the shape of the 
curves shows variability from day to day, resulting in slight changes in sensitivity and different correlations to calculate the concentration of the samples. Differences arising from the use of different calibrations are difficult to justify, so there is an urgent need for a reference to examine this and all factors influencing gluten determination.

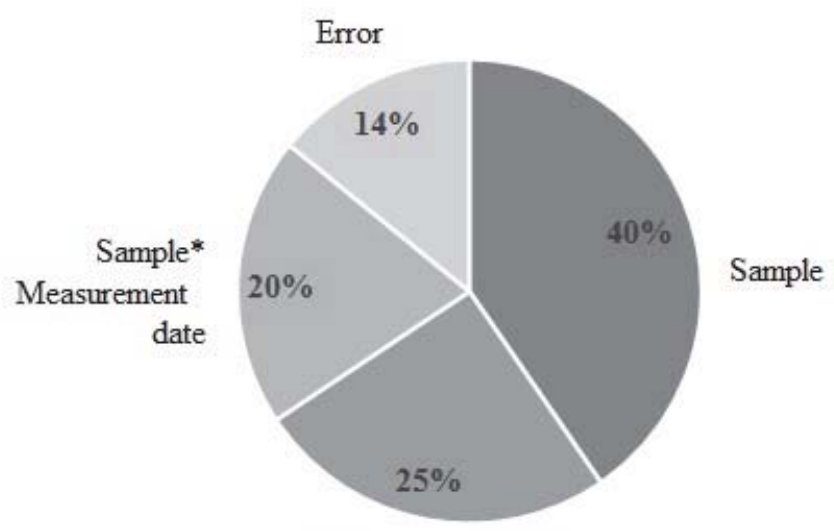

Measurement date

Fig. 3. Contribution of factors and their interactions to the standard deviation of gliadin recovery values

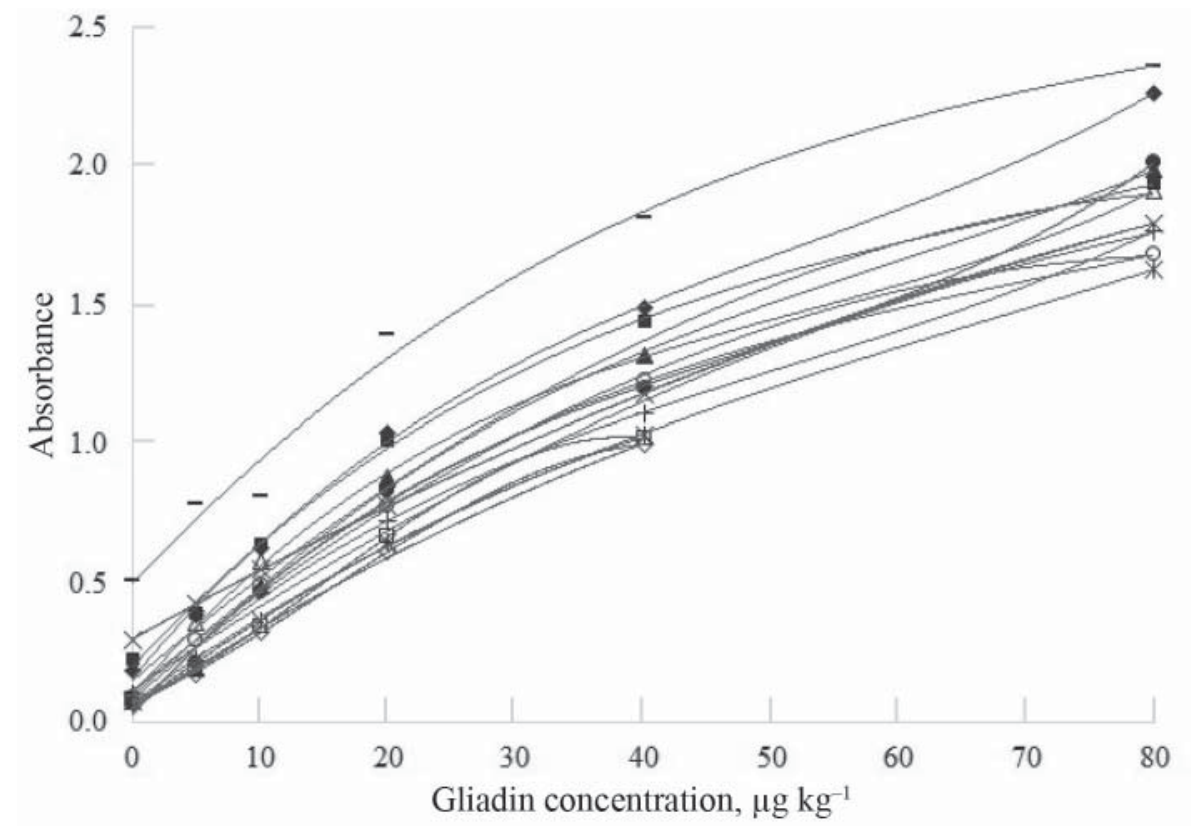

Fig. 4. Summary of calibrations on different measurement days by method "A"

ㅁ: 24.02.2016; : 03.03.2016; $\mathbf{\Delta}: 08.03 .2016 ; \bullet: 11.03 .2016 ;$ *: $25.05 .2016 ;$ —: $31.01 .2017 ; \times: 28.05 .2017$ +: 22.06.2017; $\square: 29.11 .2017 ; \diamond: 30.11 .2017 ; \Delta: 09.02 .2018 ; \bigcirc: 17.07 .2018$ 


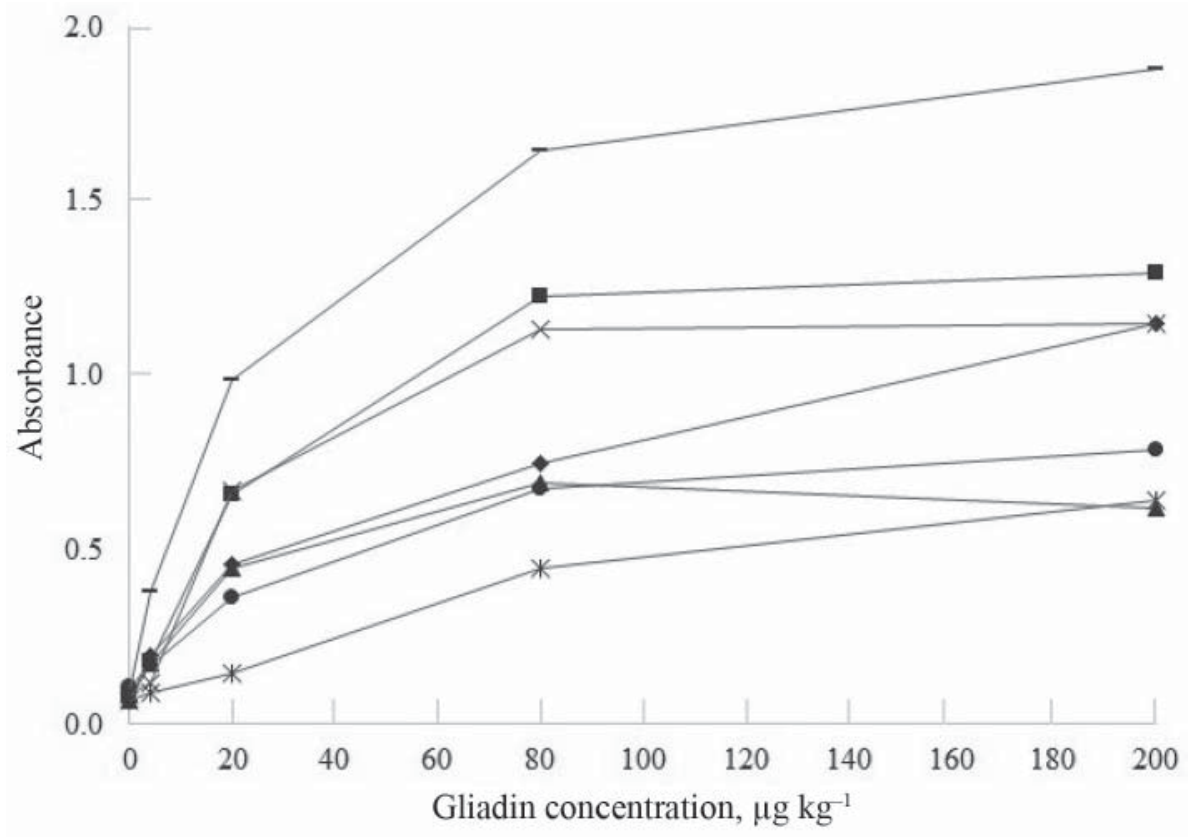

Fig. 5. Summary of calibrations on different measurement days by method "B"

17.03.2016; $>$ : 19.03.2016; $\mathbf{\Delta}: 30.05 .2016 ; \bullet: 30.01 .2017 ;$ *: 30.05.2017; —: 15.12.2017; ×: 01.08 .2018

\subsection{Examining factors influencing precision of the methods}

Although the repeatability values are acceptable for a general ELISA method (Aввотт et al., 2010), the relative standard deviation (RSD) values of the parallel samples on the different measurement days move on a wide scale, from 0 to $15 \%$ (Fig. 6). In case of Capo sample, we can see a slight increase in the measurement uncertainty measured by method " $\mathrm{A}$ ", which can be related to the LOT number, too. However, this is not found in the results of Glenlea sample measured by ELISA kits with the same LOT number. A clear increasing tendency cannot be observed in the results of the other method, in fact, in case of Glenlea, this is rather a slight decrease (Fig. 6).

The inhomogeneity of ELISA plate may have an impact on measurement uncertainty. Based on the experiment of different locations on ELISA plate, the uncertainty of the randomly placed samples is significantly higher than the samples next to each other, so the placement could affect the RSD values (Fig. 2).

Since no significant differences can be obtained between the methods, the operators, and the samples, these factors have no significant effect on measurement uncertainty, but the inhomogeneity of the plates and the instability of the kits may affect the uncertainty of the measurements. The inhomogeneity of the samples cannot be identified analysing the RSD values of the measurements (Fig. 6).

Random errors in sample preparation could affect the repeatability values of the methods. Since neither method-dependent nor person-dependent changes can be identified, it is worth looking at the values of the calibration standards here, too. The standard solutions are readyfor-use in the kits, therefore, when evaluating their values, we can get information on the 
measurement regardless of sample preparation. We have found earlier that the repeatability of the methods is about $8 \%$. The uncertainty of measuring the calibration solutions is about $7 \%$ for both methods, so regardless of sample preparation, $7 \%$ uncertainty can occur. This result is reassuring since it can be assumed that in our special field of research, we do not cause a large measurement error during the preparation of these samples with high gluten content.

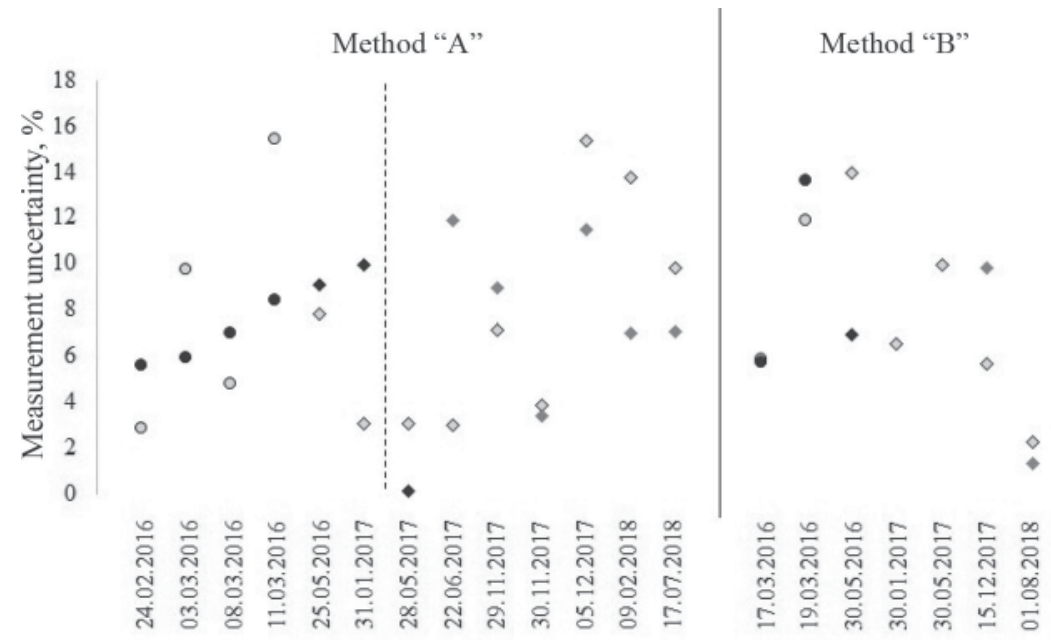

Fig. 6. Measurement uncertainty values of the three samples as a function of the measurement day measured by both methods (values before the dotted line belong to the results measured by ELISA kits with the same LOT number)

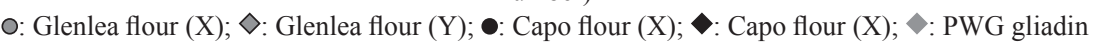

\section{Conclusions}

During our research we studied different factors influencing analytical performance of ELISA method on a special research field, where samples with high gluten content were investigated. The recovery values for accuracy and RSD values for long-term precision are acceptable for general ELISA methods. On the other hand, we pointed out that the comparison of individual analytical results of the same sample in a long-term investigation study can show much higher diversity than the mentioned parameters of the analytical performance of both ELISA methods we used. The traditional sources of analytical errors (such as sample preparation, sample placement on plate, sample stability, etc.) have no significant effects, which means both methods we used are working reliably on our samples. One of the two significant effects belongs to the sample identity, which is good as we want to compare wheat varieties as one of the main goals in our RM-oriented research. The second significant factor was the measurement days - including calibration procedures. These results reinforce also the necessity of a reference material, which allows to improve the calibration procedures, support the validation process, and give a chance to compare the analytical results obtained by different methods such as different ELISAs or even HPLC-MS. 
The authors gratefully acknowledge the contribution of Prof. Regine Schönlechner (University of Natural Resources and Applied Life Sciences) and Dr. Terry Koerner (Bureau of Chemical Safety, Health Canada) in providing the wheat samples. The authors wish to thank Dr. Katharina Scherf (Leibniz-Institute for Food Systems Biology at the Technical University of Munich) for the RP-HPLC measurement results. This work was supported by the BMEBiotechnology FIKP grant of EMMI (BME FIKP-BIO).

\section{References}

Abbott, M., Hayward, S., Ross, W., Godefroy, S.B., Ulberth, F., ... \& Delahaunt, P. (2010): Validation procedures for quantitative food allergen ELISA methods: Community guidance and best practices. J. AOAC Int., 93, $442-450$.

Besler, M., Steinhart, H. \& Paschke, A. (2001): Stability of food allergens and allergenicity of processed foods. J. Chromatogr., 756, 207-228.

Bugyi, Z., Török, K., Hajas, L., Adonyi, Z., Popping, B. \& TömösköZi, S. (2013): Comparative study of commercially available gluten ELISA kits using an incurred reference material. Qual. Assur. Saf. Crop., 5, 79-87.

Codex Alimentarius (1979): Codex standard for foods for special dietary use for persons intolerant to gluten. Codex Alimentarius Commission, Rome, Italy, STAN 118-1979, revised: 2008, amendment: 2015.

Diaz-Amigo, C. \& Popping, B. (2013): Accuracy of ELISA detection methods for gluten and reference materials: A realistic assessment. J. Agr. Food Chem., 61, 5681-5688.

Hajas, L., Scherf, K.A., Bugyi, Zs., TöröK, K., Schall, E., .. \& \& TömösKözi, S. (2016): ELISA response and gliadin composition of different wheat cultivars grown in multiple harvest years. Acta Alimentaria, 46, 187-195.

Hajas, L., Scherf, K.A., Török, K., Bugyi, Zs., Schall, E., ... \& Tömösközi, S. (2018): Variation in protein composition among wheat (Triticum aestivum L.) cultivars to identify cultivars suitable as reference material for wheat gluten analysis. Food Chem., 267, 387-394.

Ludvigsson, J.F., Leffler, D.A., Bai, J.C., Biagi, F., Fasano, A., ... \& Ciacci, C. (2013): The Oslo definitions for coeliac disease and related terms. Gut, 62, 43-52.

Hungarian Standard (1989): Edible, fodder and industrial seeds and husked products. Quality tests. Production of wheat flour in laboratory. Hungarian Standard Institution. MSZ 6367-9:1989

SCHERF, K.A. (2017): Gluten analysis of wheat starches with seven commercial ELISA test kits - Up to six different values. Food Anal. Method., 10, 234-246.

Scherf, K.A. \& Poms, R.E. (2016): Recent developments in analytical methods for tracing gluten. J. Cereal Sci., 67, $112-122$.

Schopf, M. \& Scherf, K.A. (2018): Wheat cultivar and species influence variability of gluten ELISA analyses based on polyclonal and monoclonal antibodies R5 and G12. J. Cereal Sci., 83, 32-41.

TöröK, K., Hajas, L., Bugyi, Zs., BalÁzs, G. \& TömÖsкÖZI, S. (2015): Investigation of the effects of food processing and matrix components on the analytical results of ELISA using an incurred gliadin reference material candidate. Acta Alimentaria, 44, 390-399.

van Eckert, R., Berghofer, E., Ciclitira, P.J., Chirdo, F., Denery-Papini, S., .. \& W Wieser, H. (2006): Towards a new gliadin reference material - isolation and characterisation. J. Cereal Sci., 43, 331-341.

Wieser, H., Antes, S. \& SeILmeier, W. (1998): Quantitative determination of gluten protein types in wheat flour by reversed-phase high-performance liquid chromatography. Cereal Chem., 75, 644-650.

Open Acces statement. This is an open-access article distributed under the terms of the Creative Commons Attribution 4.0 International License (https://creativecommons.org/licenses/by/4.0/), which permits unrestricted use, distribution, and reproduction in any medium, provided the original author and source are credited, a link to the $\mathrm{CC}$ License is provided, and changes - if any - are indicated. (SID_1) 